Research Paper

\title{
Lipid emulsion inhibits the vasodilation induced by a toxic dose of amlodipine in isolated rat aortae
}

\author{
Seong-Ho Ok,1,6\# Soo Hee Lee, ${ }^{2 \#}$ Ji-Yoon Kim, ${ }^{3}$ Hyun-Jin Kim, ${ }^{4,5}$ Sung Il Bae, 3 Yeran Hwang, 3 Seongyeong \\ $\mathrm{Tak}^{3}$ and Ju-Tae Sohn ${ }^{2,6}$ \\ 1. Department of Anesthesiology and Pain Medicine, Gyeongsang National University Changwon Hospital, Changwon, 51427, Republic of Korea; \\ 2. Department of Anesthesiology and Pain Medicine, Gyeongsang National University College of Medicine, Gyeongsang National University Hospital, 15 \\ Jinju-daero 816 beon-gil, Jinju-si, Gyeongsangnam-do, 52727, Republic of Korea: \\ 3. Department of Anesthesiology and Pain Medicine, Gyeongsang National University Hospital, 15 Jinju-daero 816 beon-gil, Jinju-si, Gyeongsangnam-do, \\ 52727, Republic of Korea; \\ 4. Division of Applied Life Sciences (BK21 plus), Gyeongsang National University, Gyeongsang, Republic of Korea; \\ 5. Department of Food Science \& Technology, Institute of Agriculture and Life Science, Gyeongsang National University, Gyeongsang, Republic of Korea; \\ 6. Institute of Health Sciences, Gyeongsang National University, Jinju-si, 52727, Republic of Korea. \\ \#These authors contributed equally to the work. \\ $\square$ Corresponding author: Ju-Tae Sohn, Department of Anesthesiology and Pain Medicine, Gyeongsang National University Hospital, 79 Gangnam-ro, Jinju-si, \\ 52727, Republic of Korea. E-mail: jtsohn@gnu.ac.kr; Tel.: +82-55-750-8586; Fax: +82-55-750-8142
}

(1) The author(s). This is an open access article distributed under the terms of the Creative Commons Attribution License (https://creativecommons.org/licenses/by/4.0/). See http://ivyspring.com/terms for full terms and conditions.

Received: 2019.07.17; Accepted: 2019.10.29; Published: 2019.11.09

\begin{abstract}
The goal of this study was to examine the effect of lipid emulsion on the vasodilation induced in isolated endothelium-denuded rat aortae by a toxic dose of amlodipine. We examined the effects of lipid emulsion and verapamil on amlodipine-induced vasodilation. We also examined the effects of a mixture of lipid emulsion and amlodipine, as well as the centrifuged aqueous extract (CAE) obtained by ultracentrifuging such a mixture and then removing the upper lipid layer, on amlodipine-induced vasodilation. The effect of lipid emulsion on the amlodipine concentration was examined. Lipid emulsion attenuated amlodipine-induced vasodilation in isolated aortae. Both CAE and lipid emulsion containing amlodipine inhibited amlodipine-induced vasodilation. However, there was no significant difference in amlodipine-induced vasodilation between aortae treated with CAE and those treated with lipid emulsion containing amlodipine. Verapamil inhibited amlodipine-induced vasodilation. Lipid emulsion decreased the concentration of amlodipine. Lipid emulsion attenuated the vasodilation induced by a toxic amlodipine dose in $\mathrm{NaF}$-precontracted aortae. The data show that lipid emulsion inhibited the vasodilation induced by a toxic amlodipine dose in isolated rat aortae by reducing the concentration of amlodipine. Amlodipine-induced vasodilation seems to be mediated mainly by blockade of L-type calcium channels and partially by inhibition of the Rho-kinase pathway.
\end{abstract}

Key words: amlodipine, lipid emulsion, centrifuged aqueous extract, vasodilation, verapamil

\section{Introduction}

Clinical case reports and reviews describe the effective use of lipid emulsion in treating cardiovascular depression and cardiac arrhythmia induced by toxic doses of local anesthetics or other drugs with high lipid solubility [1-3]. Lipid emulsion alleviates cardiovascular collapse induced by toxic doses of calcium channel blockers, such as verapamil [4-7]. In addition, lipid emulsion can treat severe intractable cardiovascular depression caused by toxic doses of amlodipine, which is a dihydropyridine L-type calcium channel blocker and an antihypertensive drug $[8,9]$. The proposed underlying mechanism of lipid emulsion treatment includes the scavenging effect, the inotropic effect, fatty acid supply, the attenuation of mitochondrial dysfunction, the inhibition of nitric oxide release and the 
attenuation of sodium channel blockade $[1,10]$. The scavenging effect is widely accepted to be among the underlying mechanisms associated with the lipid emulsion treatment of local anesthetic systemic toxicity $[10,11]$. This scavenging effect, which involves lipid sinks and shuttles, implies that highly lipid-soluble drugs (log [octanol/water partition coefficient]: > 2) are absorbed into the lipid phase of lipid emulsions and then transported into detoxifying and storage organs such as the liver and muscles $[10,11]$. Centrifuged aqueous extract (CAE), the lower layer obtained after ultracentrifugation of a mixture of lipid emulsion and the highly lipid-soluble local anesthetic bupivacaine, attenuates the decreased blood pressure and sodium channel blockade induced by bupivacaine alone $[12,13]$. In the case of vasodilation induced by a toxic dose of calcium channel blockers, the upper lipid layer produced by ultracentrifugation of the mixture of the calcium channel blocker and lipid emulsion seems to sequester a greater proportion of a lipid-soluble drug (verapamil) than of a less lipid-soluble drug (diltiazem), which decreases the amount of verapamil in the CAE and consequently the vasodilation induced by CAE compared with verapamil alone in Krebs solution [14-16]. However, the effect of lipid emulsion on the vasodilation induced by a toxic dose of amlodipine remains unknown. Therefore, based on previous reports, as amlodipine is relatively lipid soluble (log [octanol/water partition coefficient]: 3.0) and a calcium channel blocker, we hypothesized that lipid emulsion inhibits the vasodilation induced by a toxic dose of amlodipine by decreasing the concentration of the drug [14-17]. The objectives of this study were to examine the effect of lipid emulsion (Intralipid), which contains 100\% long-chain fatty acid, on the vasodilation induced by a toxic dose of amlodipine in isolated rat aortae and to investigate its underlying mechanism and the cellular signaling pathway of amlodipine-induced vasodilation.

\section{Materials and Methods}

Approval for all the experimental protocols was obtained from the Institutional Animal Care and Use Committee of Gyeongsang National University. All the experimental procedures and protocols were conducted according to the Guide for the Care and Use of Laboratory Animals stipulated by the Institute for Laboratory Animal Research.

\section{Preparation of isolated rat aortae followed by isometric tension measurement}

Isolated rat thoracic aortae were prepared for measurements of isometric tension as previously described by our laboratory [18]. Carbon dioxide
$(100 \%)$ was used to euthanize male Sprague-Dawley rats (body weight: 250-300 g). The descending thoracic aorta of each rat was dissected out and removed from the thorax. The isolated descending thoracic aorta was bathed in Krebs solution. Krebs solution contains the following components (mM): sodium chloride (118), glucose (11), sodium bicarbonate (25), potassium chloride (4.7), calcium chloride (2.4), magnesium sulfate (1.2) and monopotassium phosphate (1.2). The periaortic tissues containing connective tissue and fat were dissected and removed under a microscope. The aorta was cut into an aortic ring approximately 2.5 $\mathrm{mm}$ long. The endothelium of the aortic ring was removed by inserting 25-gauge needles into the lumen of the isolated aorta and rolling the vessel back and forth for a few seconds. Isolated aortic rings were suspended in a Grass isometric transducer (FT-03, Grass Instruments, Quincy, MA, USA) attached to an organ bath containing $10 \mathrm{~mL}$ of Krebs solution maintained at $37^{\circ} \mathrm{C}$. A baseline resting tension of $3.0 \mathrm{~g}$ was maintained for $120 \mathrm{~min}$ to reach equilibrium, and the Krebs solution was exchanged approximately every $30 \mathrm{~min}[18,19]$. A mix of $95 \%$ oxygen gas and $5 \%$ carbon dioxide gas was supplied to aerate the Krebs solution in the organ bath to maintain a $\mathrm{pH}$ of approximately 7.4. To confirm endothelial denudation of the isolated rat aortae, we added phenylephrine $\left(10^{-8} \mathrm{M}\right)$ into the organ bath containing the aortae. After phenylephrine $\left(10^{-8} \mathrm{M}\right)$ produced a sustained and stable concentration, we added acetylcholine $\left(10^{-5}\right.$ M) into the organ bath to verify endothelial denudation; any aorta that relaxed by less than $15 \%$ in response to acetylcholine was considered to be denuded of its endothelium. After the aortic rings showing acetylcholine-induced relaxation were washed with fresh Krebs solution, baseline resting tension was recovered. Then, contraction of some isolated endothelium-denuded rat aortic rings was induced by isotonic $60 \mathrm{mM} \mathrm{KCl}$, and the magnitude of the resulting contraction was used as a reference value to express the magnitude of norepinephrine-induced contraction. Next, the isotonic $60 \mathrm{mM} \mathrm{KCl}$ solution was washed away with fresh Krebs solution, and baseline resting tension was restored. Then, we performed the following experimental protocols. As amlodipine induces the nitric oxide-dependent relaxation of isolated aorta and lipid emulsion attenuates acetylcholine-induced nitric oxide-dependent relaxation, the isolated endothelium-denuded aortic rings used for the following experimental protocols were pretreated with the nitric oxide synthase inhibitor $\mathrm{NW}^{\mathrm{W}}$-nitro-L-arginine methyl ester (L-NAME, $10^{-4} \mathrm{M}$ ) to avoid the confounding factor of nitric oxide release from any residual endothelium; the abovementioned 
two factors hypothetically might complicate the interpretation of results regarding the effect of lipid emulsion on amlodipine-induced vasodilation in the current study $[20,21]$.

\section{Experimental protocol}

First, we examined the effect of cotreatment with lipid emulsion on the vasodilation induced by toxic concentrations of amlodipine in isolated endothelium-denuded rat aortae precontracted with norepinephrine. Norepinephrine $\left(10^{-6} \mathrm{M}\right)$ was added to the organ bath to produce sustained and stable vasoconstriction. Then, a toxic dose of amlodipine $(3 \times$ $\left.10^{-7} \mathrm{M}\right)$ alone or combined with lipid emulsion (0.25 and $1 \%$ ) was added to produce vasodilation in isolated endothelium-denuded rat aortae precontracted with norepinephrine [22]. Amlodipine-induced vasodilation was monitored for $70 \mathrm{~min}$ in the presence and absence of lipid emulsion. In addition, we examined the effect of posttreatment with lipid emulsion on the vasodilation induced by amlodipine in the endothelium-denuded rat aorta precontracted with $\mathrm{KCl}$. After contraction induced by isotonic $60 \mathrm{mM} \mathrm{KCl}$ solution reached a plateau in the aortic rings, amlodipine $\left(10^{-7} \mathrm{M}\right)$ was added to the organ bath. After amlodipine-induced vasodilation reached approximately $20 \%$ vasodilation with respect to the previous contraction induced by the $\mathrm{KCl}$ solution, some aortic rings were treated with lipid emulsion (0.3 and 1\%). Then, amlodipine-induced vasodilation was monitored for $70 \mathrm{~min}$ in the presence or absence of lipid emulsion. Additionally, we compared the magnitude of amlodipine-induced vasodilation for $70 \mathrm{~min}$ in isolated rat aorta precontracted with norepinephrine $\left(10^{-6} \mathrm{M}\right)$ or $60 \mathrm{mM}$ $\mathrm{KCl}$.

Second, based on a previous report that lipid emulsion attenuates the vasodilation induced by toxic doses of highly lipid-soluble calcium channel blockers (bepridil and verapamil) by reducing the concentrations of these drugs in the CAE because larger amounts of these drugs partition into the upper lipid layer compared with the less lipid-soluble calcium channel blocker diltiazem, we examined the effects of a mixture of lipid emulsion and amlodipine as well as CAE obtained by ultracentrifuging such a mixture on isolated endothelium-denuded rat aortae precontracted with norepinephrine [16]. CAE, which is used in this experimental protocol, was obtained from the ultracentrifugation of the mixture of lipid emulsion plus amlodipine and the subsequent removal of the upper lipid layer (Fig. 1). After norepinephrine $\left(10^{-6} \mathrm{M}\right)$ produced a sustained and stable contraction, CAE or amlodipine with or without lipid emulsion was added to the organ bath to produce amlodipine-induced vasodilation in the precontracted aortae, and then amlodipine-induced vasodilation was monitored for $70 \mathrm{~min}$. Lipid emulsion containing amlodipine and CAE were produced using the following methods. Lipid emulsion $(20 \%) 10 \mathrm{~mL}$ and amlodipine $\left(6 \times 10^{-3} \mathrm{M}\right) 10$ $\mu \mathrm{L}$ were mixed using a Mylab Multi Mixer (\#SLRM-3, Seoulin Bioscience, Bundang, Korea) at 60 RPM for 30 min to produce approximately $20 \%$ lipid emulsion containing approximately $6 \times 10^{-6} \mathrm{M}$ amlodipine (Fig. 1A). A $500 \mu \mathrm{L}$ volume of the resulting mixture was added to the organ bath with $9.5 \mathrm{~mL}$ Krebs solution so that the bath contained 1\% lipid emulsion and $3 \times 10^{-7}$ $\mathrm{M}$ amlodipine. Approximately 20\% lipid emulsion containing approximately $6 \times 10^{-6} \mathrm{M}$ amlodipine was ultracentrifuged at $4^{\circ} \mathrm{C}$ and $75,000 \mathrm{~g}$ for $12 \mathrm{~min}$ using an Optima ${ }^{\mathrm{TM}}$ MAX-XP ultracentrifuge (Beckman Coulter Life Sciences, Indianapolis, IN, USA) to separate the emulsion into two layers: a lower CAE layer (Fig. 1B) and an upper lipid layer (Fig. 1B). Five hundred microliters of this lower layer (Fig. 1B) was added to the organ bath with $9.5 \mathrm{~mL}$ Krebs solution. The resulting CAE corresponded to $1 \%$ lipid emulsion and $3 \times 10^{-7} \mathrm{M}$ amlodipine in the organ bath. A $490 \mu \mathrm{L}$ volume of Krebs solution and a $10 \mu \mathrm{L}$ volume of amlodipine $\left(3 \times 10^{-4} \mathrm{M}\right)$ were added to the organ bath with $9.5 \mathrm{~mL}$ Krebs solution so that the amlodipine (at a final concentration of $3 \times 10^{-7} \mathrm{M}$ ) would induce vasodilation. We examined the effect of pretreatment with verapamil on amlodipine-induced vasodilation in isolated endothelium-denuded rat aortae to confirm that amlodipine-induced vasodilation involves the blockade of L-type calcium channels [23]. After endothelium-denuded rat aortae were pretreated with verapamil $\left(10^{-5} \mathrm{M}\right)$ for $20 \mathrm{~min}$, norepinephrine $\left(10^{-6} \mathrm{M}\right)$ was added to the organ bath to produce vasoconstriction in the presence or absence of verapamil. Then, amlodipine $\left(3 \times 10^{-7} \mathrm{M}\right)$ was added to the organ bath to produce vasodilation in the presence or absence of verapamil. For comparison with amlodipine-induced vasodilation in the presence of verapamil, some endothelium-denuded aortic rings were not posttreated with amlodipine, and vasodilation was induced by verapamil alone $\left(10^{-5} \mathrm{M}\right)$. The vasodilation induced by amlodipine or verapamil alone and combined treatment with verapamil and amlodipine was monitored for $70 \mathrm{~min}$ and compared.

Third, we examined the effects of the Rho-kinase inhibitor Y-27632 (10-6 to $\left.10^{-5} \mathrm{M}\right)$ and the protein kinase C (PKC) inhibitor GF109203X $\left(10^{-6}\right.$ to $\left.10^{-5} \mathrm{M}\right)$ on the contraction induced by norepinephrine $\left(10^{-9}\right.$ to $10^{-5}$ $\mathrm{M})$ in isolated endothelium-denuded rat aortae. Isolated endothelium-denuded rat aortic rings were pretreated with Y-27632 (10-6 to $\left.10^{-5} \mathrm{M}\right)$ and 
GF109203X (10-6 to $\left.10^{-5} \mathrm{M}\right)$ for $20 \mathrm{~min}$. Then, norepinephrine $\left(10^{-9}\right.$ to $\left.10^{-5} \mathrm{M}\right)$ was cumulatively added to the organ bath to produce a norepinephrine concentration-response curve in the presence or absence of Y-27632 or GF109203X.

Fourth, as the precontraction induced by norepinephrine involves the PKC- and Rho-kinase-mediated pathway, as demonstrated by the results of the experimental protocol above, we examined the effect of pretreatment with lipid emulsion on the vasodilation induced by amlodipine in isolated endothelium-denuded rat aortae precontracted with the Rho-kinase activator sodium fluoride $(\mathrm{NaF})$ or the PKC activator phorbol 12,13-dibutyrate (PDBu) [23]. Endothelium-denuded rat aortae were pretreated with lipid emulsion $(0.25$ and $1 \%)$ for $20 \mathrm{~min}$. Then, $\mathrm{NaF}\left(3 \times 10^{-8} \mathrm{M}\right)$ or PDBu $\left(10^{-6} \mathrm{M}\right)$ was added to the organ bath to produce a sustained and stable contraction in the presence or absence of lipid emulsion. Then, amlodipine $\left(3 \times 10^{-7}\right.$ M) was added to the organ bath to produce vasodilation. Amlodipine-induced vasodilation was monitored for $70 \mathrm{~min}$. In addition, we compared the effects of $\mathrm{NaF}$ and PDBu on amlodipine $\left(3 \times 10^{-7}\right.$ $\mathrm{M}$ )-induced vasodilation for $70 \mathrm{~min}$ to investigate the underlying mechanism of the amlodipine-induced vasodilation of isolated aortae.

\section{Effect of lipid emulsion on the amlodipine concentration in Krebs solution}

Amlodipine $\left(3 \times 10^{-7} \mathrm{M}\right)$ in Krebs solution was mixed with Intralipid ( 0.25 and $1 \%)$ with a rotator for 30 min to emulsify the amlodipine and lipid emulsion as previously described $[16,24]$. After centrifugation at $75,000 \mathrm{~g}$ for $40 \mathrm{~min}$, the amlodipine in the aqueous phase, which is equivalent to CAE, was measured by ultraperformance liquid chromatography-quadrupole time-of-flight mass spectrometry (UPLC-Q-TOF MS; Waters, Milford, MA, USA). The aqueous layer was injected into an Acquity UPLC BEH $\mathrm{C}_{18}$ column $(100 \times$ $2.1 \mu \mathrm{mm}, 1.7 \mu \mathrm{m}$; Waters) equilibrated with water/acetonitrile (95:5) containing $0.1 \%$ formic acid and eluted with a linear gradient $(5-100 \%)$ of acetonitrile containing $0.1 \%$ formic acid at a flow rate of $0.35 \mathrm{~mL} / \mathrm{min}$ for $7 \mathrm{~min}$. The eluted amlodipine was analyzed by Q-TOF MS (Waters) in positive electrospray ionization (ESI) mode and identified by the precursor and product ions of $\mathrm{m} / \mathrm{z} 289.21$ and 140.13 , respectively, in multiple reaction monitoring. The capillary and sampling cone voltages were set at 3 $\mathrm{kV}$ and $30 \mathrm{~V}$, respectively. The desolvation temperature and flow rate were $100^{\circ} \mathrm{C}$ and $800 \mathrm{~L} / \mathrm{h}$, respectively. The source temperature was set at $400^{\circ} \mathrm{C}$. LockSpray with leucine-enkephalin $([\mathrm{M}+\mathrm{H}]=556.2771$ $\mathrm{Da})$ was used at a frequency of 10 seconds to ensure the reproducibility and accuracy of all analyses. All mass data were collected and analyzed by UIFI 1.8.2 (Waters).

\section{Materials}

Norepinephrine, $\mathrm{KCl}$, amlodipine, $\mathrm{PDBu}, \mathrm{NaF}$, GF109203X, L-NAME, verapamil, phenylephrine and acetylcholine were obtained from Sigma Aldrich (St. Louis, MO, USA). Y-27632 was obtained from Calbiochem (La Jolla, CA, USA). Intralipid ${ }^{\circledR}(20 \%)$ was obtained from Fresenius Kabi Korea (Seoul, Korea). All chemical concentrations are expressed as the final molar concentration in the organ bath. The concentration of lipid emulsion is expressed as the percentage in the organ bath. PDBu, GF109203X and amlodipine were dissolved in dimethyl sulfoxide (final concentration of dimethyl sulfoxide: less than $0.1 \%)$. All the chemicals except PDBu, GF109203X and amlodipine were dissolved in distilled water.

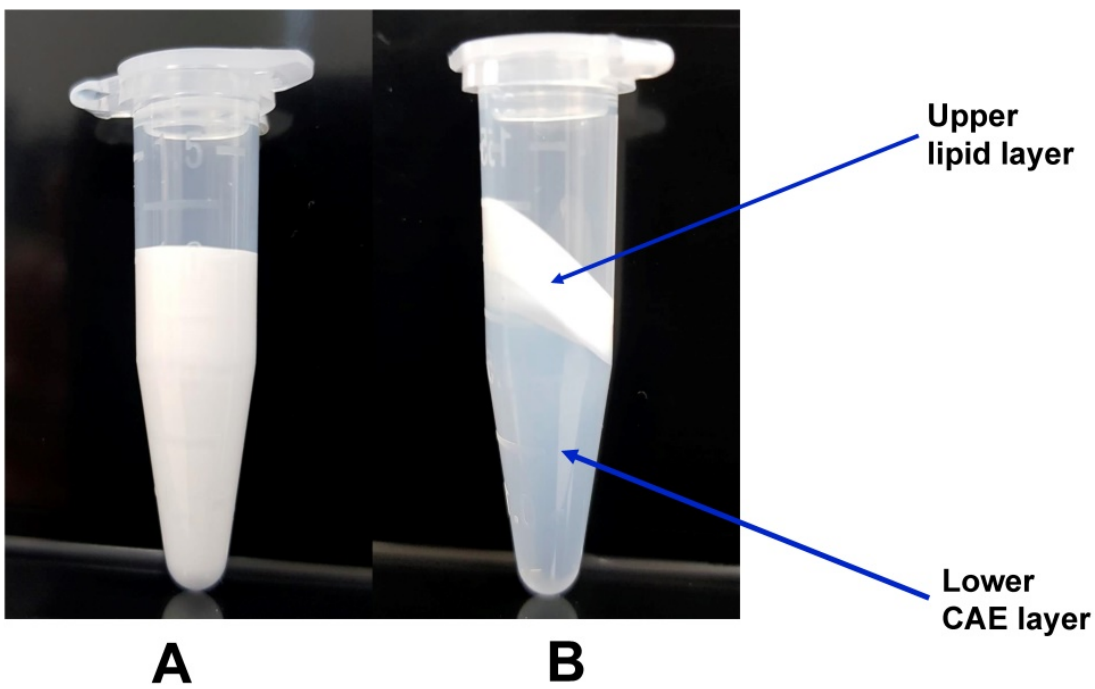

Figure 1. This figure shows the conditions before (A) and after (B) ultracentrifugation $\left(75,000 \mathrm{~g}\right.$ at $4^{\circ} \mathrm{C}$ for $\left.12 \mathrm{~min}\right)$ of approximately $20 \%$ Intralipid containing approximately $6 \times 10^{-6} \mathrm{M}$ amlodipine. Ultracentrifugation separated the mixture into the upper lipid layer and lower centrifuged aqueous extract (CAE) layer. 


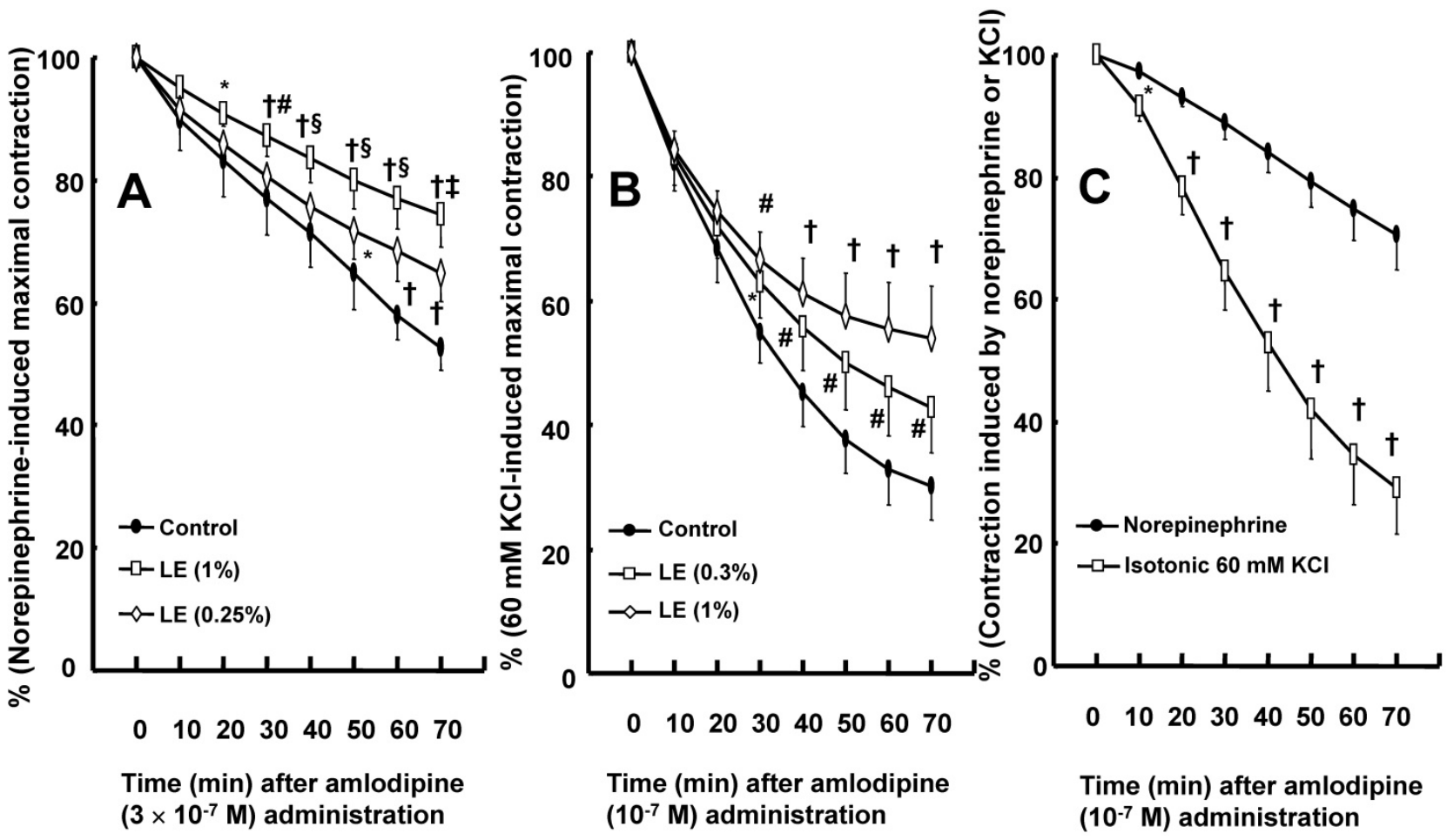

Figure 2. A: Effect of cotreatment with lipid emulsion (Intralipid, LE) on the vasodilation induced by amlodipine (3 $\left.\times 10^{-7} \mathrm{M}\right)$ in isolated endothelium-denuded rat aortae precontracted with norepinephrine $\left(10^{-6} \mathrm{M}\right)$. Data $(\mathrm{N}=6)$ are shown as the mean \pm SD and expressed as the percentage of maximal contraction induced by norepinephrine. $\mathrm{N}$ represents the number of isolated aortae. $* P<0.01$ and $+P<0.001$ versus control. \#P<0.05, $\$ P<0.01$ and $\ddagger P<0.001$ versus $0.25 \% L E$. $\mathbf{B}$ : Effect of posttreatment with LE on the vasodilation induced by amlodipine (10-7 M) in isolated endothelium-denuded rat aortae precontracted with 60 mM $\mathrm{KCl}$. After amlodipine $\left(10^{-7} \mathrm{M}\right)$ produced approximately $20 \%$ vasodilation from the contraction induced by isotonic $60 \mathrm{mM} \mathrm{KCl}$, some aortic rings were treated with $\mathrm{LE}(0.3$ and $1 \%$ ). Data (control and 1\% LE: $\mathrm{N}=5 ; 0.3 \% \mathrm{LE}: \mathrm{N}=4$ ) are shown as the mean $\pm \mathrm{SD}$ and expressed as the percentage of contraction induced by isotonic $60 \mathrm{mM} \mathrm{KCl}$. $\mathrm{N}$ represents the number of rats from which isolated aortae were obtained. $* P<0.05, \# P<0.01$ and $+P<0.001$ versus control. $\mathbf{C}$ : Amlodipine-induced vasodilation in isolated endothelium-denuded rat aortae precontracted with norepinephrine $\left(10^{-6} \mathrm{M}\right)$ or isotonic $60 \mathrm{mM} \mathrm{KCl}$. Data $(\mathrm{N}=8)$ are shown as the mean $\pm \mathrm{SD}$ and expressed as the percentage of contraction induced by $\mathrm{KCl}$ or norepinephrine. $\mathrm{N}$ represents the number of isolated rat aortae. $* P<0.05$ and $+P<0.001$ versus norepinephrine.

\section{Statistical analysis}

Data are expressed as the mean \pm SD or median \pm interquartile range. The vasodilation induced by amlodipine is expressed as the percentage of contraction induced by norepinephrine, $\mathrm{KCl}, \mathrm{PDBu}$ and $\mathrm{NaF}$. The effects of lipid emulsion, CAE and verapamil on amlodipine-induced vasodilation were analyzed using a generalized linear mixed-effects model (Stata version 14.2, StataCorp LP, Lakeway Drive College Station, TX, USA) [25]. The magnitude of norepinephrine-induced contraction is expressed as the percentage of maximal contraction induced by isotonic $60 \mathrm{mM} \mathrm{KCl}$. The effects of Y-27632 and GF109203X on norepinephrine-induced contraction and the effects of $\mathrm{NaF}, \mathrm{PDBu}$, norepinephrine and $\mathrm{KCl}$ on amlodipine-induced vasodilation were analyzed with a generalized linear mixed-effects model. The effect of lipid emulsion on amlodipine concentration was analyzed using Kruskal-Wallis test followed by Dunn's multiple comparison test. A $P$ value less than 0.05 was considered statistically significant.

\section{Results}

Cotreatment with lipid emulsion (0.25 and 1\%) inhibited amlodipine $\left(3 \times 10^{-7} \quad \mathrm{M}\right)$-induced vasodilation in isolated endothelium-denuded rat aortae precontracted with norepinephrine in a concentration-dependent pattern (Fig. 2A; $P<0.01$ versus control at 50 and $70 \mathrm{~min}$ ). Posttreatment with lipid emulsion (0.3 and $1 \%)$ attenuated amlodipine $\left(\begin{array}{ll}10^{-7} & \mathrm{M}\end{array}\right)$-induced vasodilation in isolated endothelium-denuded rat aortae precontracted with isotonic $60 \mathrm{mM} \mathrm{KCl}$ (Fig. 2B; $P<0.05$ versus control at 30 to $70 \mathrm{~min})$. Amlodipine $\left(10^{-7} \mathrm{M}\right)$-induced vasodilation was enhanced under $\mathrm{KCl}$-induced contraction compared with norepinephrine-induced contraction (Fig. 2C; $P<0.05$ versus 10 to $70 \mathrm{~min}$ ).

Both CAE, which corresponds to a $1 \%$ lipid emulsion containing $3 \times 10^{-7} \mathrm{M}$ amlodipine in the organ bath, and lipid emulsion (1\%) containing amlodipine $\left(3 \times 10^{-7} \mathrm{M}\right)$ inhibited amlodipine-induced vasodilation (Fig. 3A; $P<0.001$ versus amlodipine at 10 to $70 \mathrm{~min}$ ). However, CAE did not significantly inhibit amlodipine-induced vasodilation compared with lipid emulsion $(1 \%)$ containing amlodipine $(3 \times$ 
$\left.10^{-7} \mathrm{M}\right)$ (Fig. 3A). Pretreatment with verapamil $\left(10^{-5}\right.$ M) inhibited amlodipine $\left(3 \times 10^{-7} \mathrm{M}\right)$-induced vasodilation in isolated endothelium-denuded rat aortae precontracted with norepinephrine (Fig. 3B; $P$ $<0.01$ versus amlodipine alone at 20 to $70 \mathrm{~min}$ ). However, there was no significant difference between vasodilation induced by verapamil $\left(10^{-5} \mathrm{M}\right)$ alone and vasodilation induced by verapamil $\left(10^{-5} \mathrm{M}\right)$ plus

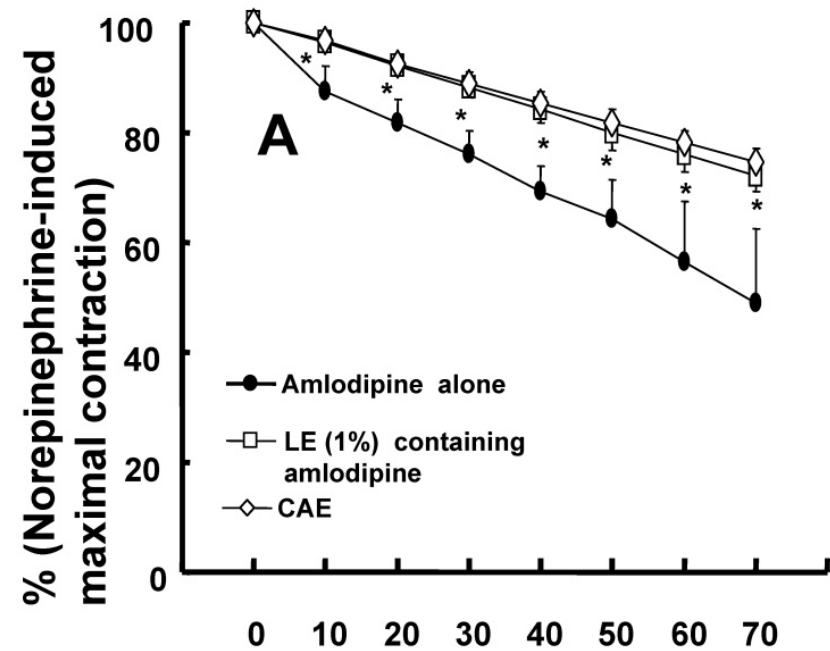

Time (min) after amlodipine $\left(3 \times 10^{-7} \mathrm{M}\right)$ administration amlodipine $\left(3 \times 10^{-7} \mathrm{M}\right)$ in isolated rat aortae precontracted with norepinephrine (Fig. 3B).

Y-27632 $\left(10^{-6}\right.$ to $\left.10^{-5} \quad \mathrm{M}\right)$ inhibited norepinephrine-induced contraction (Fig. $4 \mathrm{~A} ; 3 \times 10^{-6}$ and $10^{-5} \mathrm{M}: P<0.001$ versus control at $3 \times 10^{-9}$ to $10^{-5} \mathrm{M}$ norepinephrine), as did GF109203X $\left(3 \times 10^{-6}\right.$ and $10^{-5}$ M Fig. 4B; $P<0.001$ versus control at $3 \times 10^{-9}$ to $3 \times 10^{-8}$ M norepinephrine).

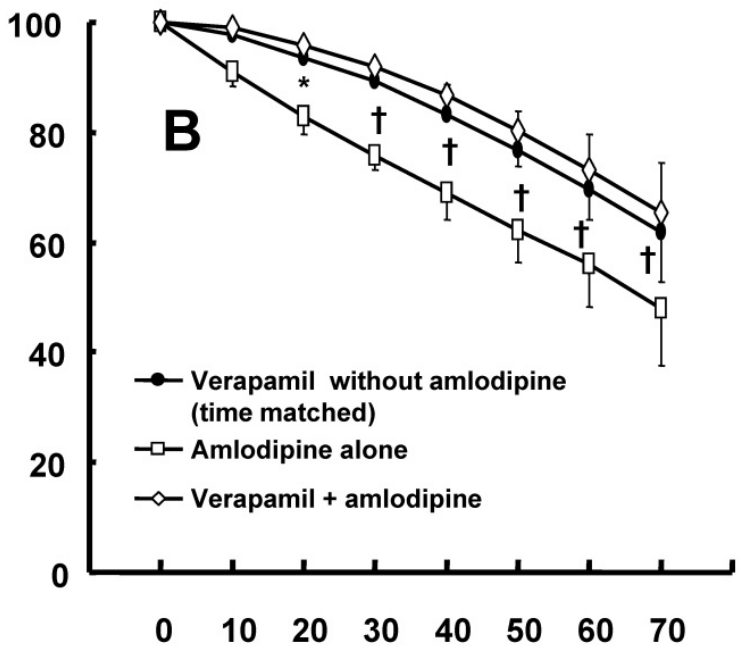

Time (min) after amlodipine $\left(3 \times 10^{-7} \mathrm{M}\right)$ administration

Figure 3. A: Effects of a mixture of lipid emulsion (Intralipid, LE: $1 \%)$ and amlodipine $\left(3 \times 10^{-7} \mathrm{M}\right)$, centrifuged aqueous extract (CAE) and amlodipine $\left(3 \times 10^{-7} \mathrm{M}\right)$ alone in isolated endothelium-denuded rat aortae precontracted with norepinephrine (10-6 M). The CAE, which was obtained by using ultracentrifugation to separate the lipid emulsion containing amlodipine into an upper lipid layer and a lower CAE layer, corresponds to LE (1\%) and amlodipine $\left(3 \times 10^{-7} \mathrm{M}\right)$ in the organ bath. Data $(\mathrm{N}=9)$ are shown as the mean $\pm \mathrm{SD}$ and expressed as the percentage of maximal contraction induced by norepinephrine. $\mathrm{N}$ represents the number of isolated rat aortae. $* P<0.001$ versus amlodipine alone. B: The vasodilation induced by amlodipine or verapamil alone and by combined treatment with verapamil and amlodipine in isolated endothelium-denuded rat aorta precontracted with norepinephrine $\left(10^{-6} \mathrm{M}\right)$. Data (verapamil without amlodipine: $\mathrm{N}=8$, amlodipine alone: $\mathrm{N}=5$, and verapamil plus amlodipine: $\mathrm{N}=8$ ) are shown as the mean \pm SD and expressed as the percentage of maximal contraction induced by norepinephrine. $\mathrm{N}$ represents the number of isolated rat aortae. $* P<0.01$ and $\dagger P<0.001$ versus amlodipine alone.
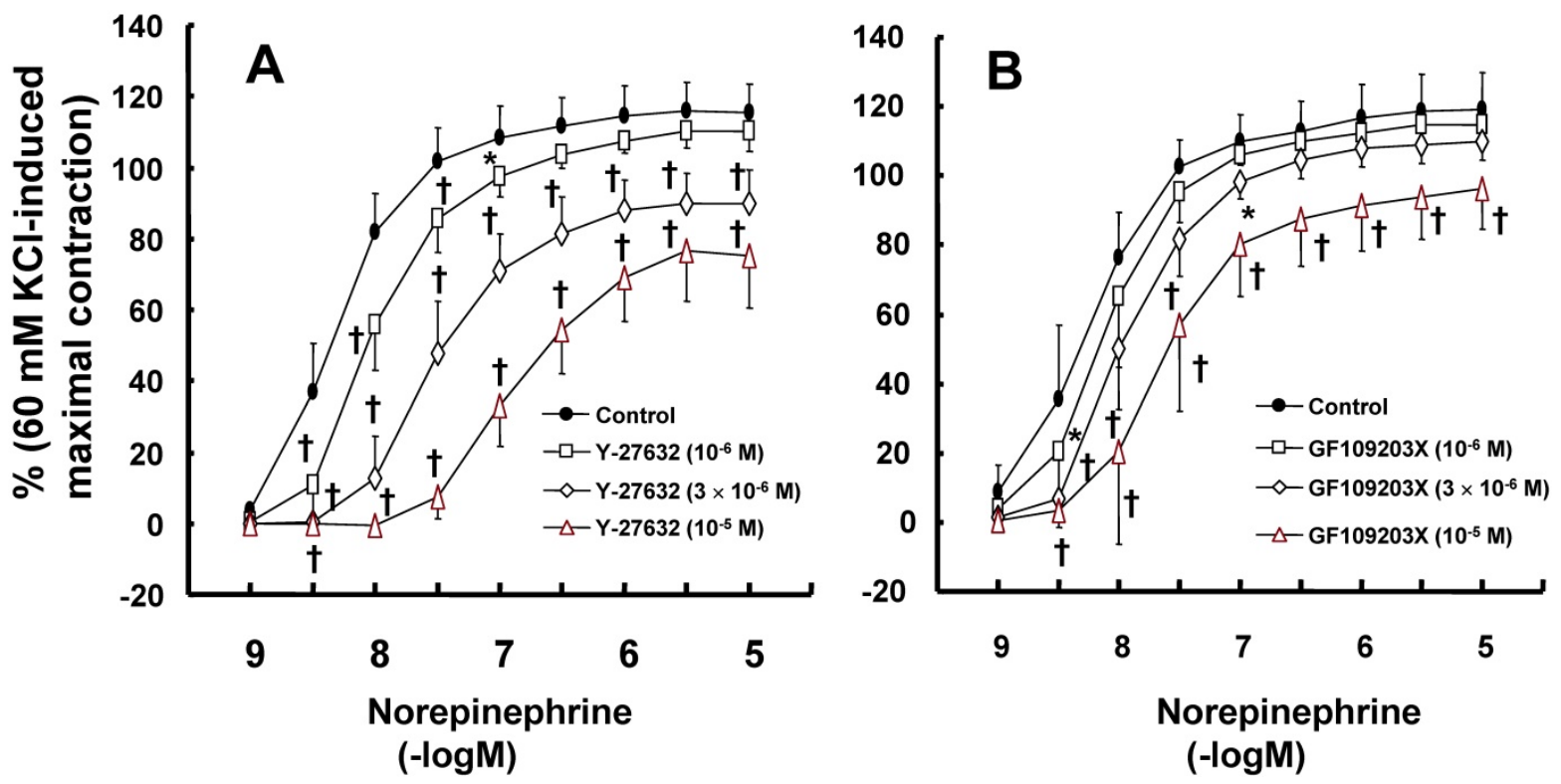

Figure 4. Effects of Rho-kinase inhibitor $Y-27632(\mathbf{A} ; \mathrm{N}=8)$ and protein kinase $\mathrm{C}$ inhibitor GF109203X (B; control: $N=8,10-6$ : $: N=7 ; 3 \times 10-6$ and $10-5$ M = 8) on the contraction induced by norepinephrine in isolated endothelium-denuded rat aortae. Data are shown as the mean \pm SD and expressed as the percentage of maximal contraction induced by $60 \mathrm{mM} \mathrm{KCl}$. $\mathrm{N}$ represents the number of isolated rat aortae. $* P<0.05$ and $\dagger P<0.001$ versus control. 
Amlodipine $\left(3 \times 10^{-7} \mathrm{M}\right)$-induced vasodilation was higher in endothelium-denuded rat aorta precontracted with $\mathrm{NaF}\left(8 \times 10^{-3} \mathrm{M}\right)$ than in those precontracted with PDBu $\left(10^{-6} \mathrm{M}\right)$ (Fig. 5A; $P<0.05$ versus PDBu at 10 to $70 \mathrm{~min})$. Lipid emulsion (1\%) attenuated amlodipine $\left(3 \times 10^{-7} \mathrm{M}\right)$-induced vasodilation in isolated endothelium-denuded rat aortae precontracted with $\mathrm{NaF}$ (Fig. 5B; $P<0.05$ versus control at 10 to $70 \mathrm{~min})$. However, lipid emulsion $(1 \%)$ slightly inhibited amlodipine $\left(3 \times 10^{-7}\right.$
M)-induced vasodilation in isolated endotheliumdenuded rat aortae precontracted with PDBu (Fig. 5C; $P<0.01$ versus control at 30 to $70 \mathrm{~min}$ ).

Lipid emulsion (0.25 and 1\%) significantly decreased the concentration of amlodipine (originally $3 \times 10^{-7}$ M; Fig. 6 ; $P<0.05$ versus amlodipine alone) in CAE, which was obtained by ultracentrifugation of the mixture of lipid emulsion and amlodipine.

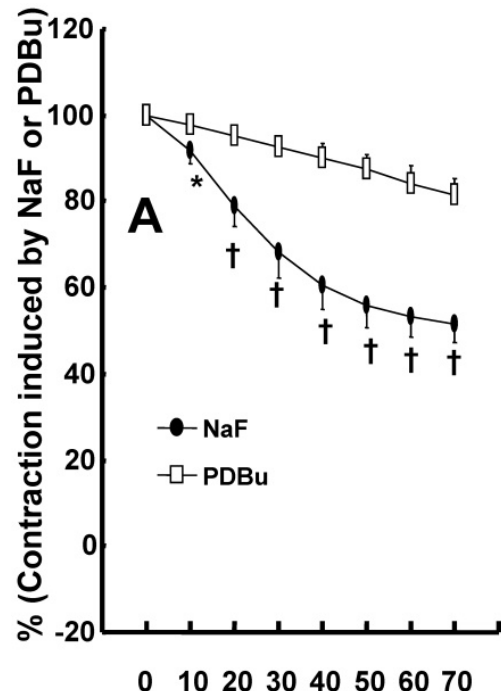

Time (min) after amlodipine administration

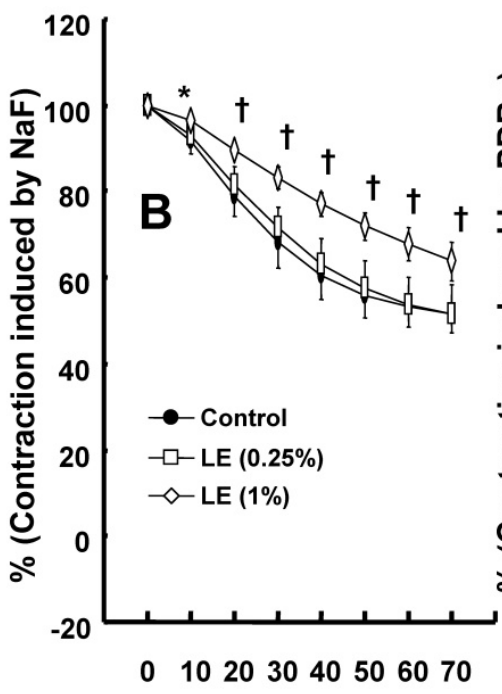

Time ( $\mathrm{min})$ after amlodipine administration

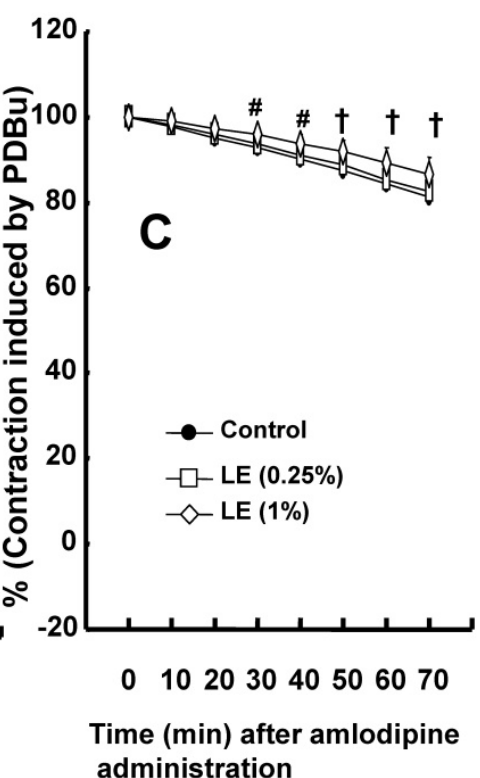

Time (min) after amlodipine administration

Figure 5. A: Amlodipine $\left(3 \times 10^{-7} \mathrm{M}\right)$-induced vasodilation in isolated endothelium-denuded rat aortae precontracted with the Rho-kinase activator sodium fluoride $\left(\mathrm{NaF}, 8 \times 10^{-3} \mathrm{M}\right)$ or the protein kinase $\mathrm{C}$ activator phorbol 12,13-dibutyrate (PDBu, 10-6 $\mathrm{M}$ ). Data $(\mathrm{N}=8)$ are shown as the mean $\pm \mathrm{SD}$ and expressed as the percentage of maximal contraction induced by $\mathrm{NaF}$ or PDBu. $\mathrm{N}$ represents the number of isolated rat aortae. $* P<0.05$ and $\dagger P<0.001$ versus PDBu. B and $\mathbf{C}$ : Effect of pretreatment with lipid emulsion (Intralipid, LE) on the vasodilation induced by amlodipine $\left(3 \times 10^{-7} \mathrm{M}\right)$ in isolated endothelium-denuded rat aorta precontracted with $\mathrm{NaF}\left(8 \times 10^{-3} \mathrm{M}, \mathrm{B}\right)$ or PDBu $\left(10^{-6} \mathrm{M}, \mathrm{C}\right)$. Data $(\mathrm{N}=8)$ are shown as the mean $\pm \mathrm{SD}$ and expressed as the percentage of maximal contraction induced by $\mathrm{NaF}$ or PDBu. $N$ represents the number of isolated rat aortae. $* P<0.05, \# P<0.01$ and $\dagger P<0.001$ versus control.

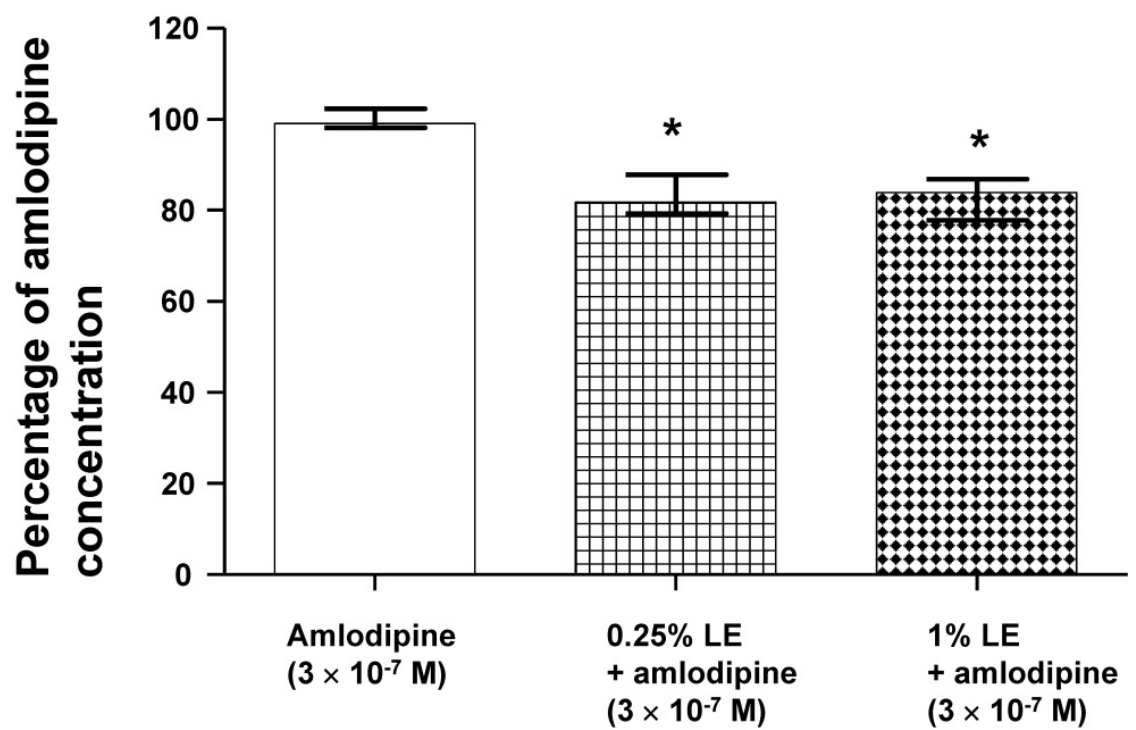

Figure 6. Effect of lipid emulsion (Intralipid, LE) on the amlodipine concentration $\left(3 \times 10^{-7} \mathrm{M}\right)$ in Krebs solution. Amlodipine concentration was measured using ultraperformance liquid chromatography-quadrupole time-of-flight mass spectrometry. Data are shown as the median \pm interquartile range and expressed as the percentage of the original amlodipine concentration $\left(3 \times 10^{-7} \mathrm{M}\right)$. Statistical analysis was performed using the Kruskal-Wallis test followed by Dunn's multiple comparison test. Experiments were repeated five times. $* P<0.05$ versus amlodipine alone. 


\section{Discussion}

This study suggests that lipid emulsion attenuates the vasodilation induced by a toxic dose of amlodipine, which is associated with the reduction in amlodipine concentrations caused by lipid emulsion. The major findings of this study are as follows: 1 ) cotreatment or posttreatment with lipid emulsion attenuated the amlodipine-induced vasodilation of isolated aortae; 2) CAE produced less vasodilation than amlodipine alone; 3) there was no significant difference between vasodilation induced by verapamil alone and vasodilation induced by combined treatment with verapamil and amlodipine; and 4) the concentration of amlodipine was decreased in the CAE (Fig. 6), which was derived from the mixture of lipid emulsion and amlodipine.

The treatment of toxicity induced by calcium channel blockers includes gastrointestinal decontamination, supportive care, vasopressors, calcium, hyperinsulinemia/euglycemia, lipid emulsion and extracorporeal membrane oxygenation [26]. Among these treatments, lipid emulsion and extracorporeal membrane oxygenation can be used to treat intractable hypotension induced by a toxic dose of amlodipine $[8,9,26,27]$. The lipid sink theory, which is popularly accepted, indicates that highly lipid-soluble drugs are absorbed into lipid emulsion from tissues including the heart and aorta $[12,13,16,28-30]$. Lipid emulsion inhibits the vasodilatory effects of calcium channel blockers including, in decreasing order of inhibition, bepridil, verapamil and nifedipine; this phenomenon seems to be associated with lipid solubility [14]. In addition, CAE, which is derived from a mixture of lipid emulsion and a relatively lipid-soluble calcium channel blocker (bepridil or verapamil) by ultracentrifugation of the mixture followed by the removal of the upper lipid layer, attenuated vasodilation induced by toxic doses of bepridil and verapamil alone but did not significantly inhibit vasodilation induced by bepridil or verapamil alone compared with a mixture of lipid emulsion and a calcium channel blocker [16]. Moreover, CAE from a mixture of the highly lipid-soluble local anesthetic bupivacaine and lipid emulsion produces a smaller decrease in mean blood pressure than bupivacaine alone [12]. CAE obtained from the mixture of lipid emulsion and bupivacaine partially reverses the sodium current blockade induced by bupivacaine alone [13]. These previous studies suggest that when a lower CAE layer and an upper lipid layer from the mixture of lipid emulsion with lipid-soluble drugs are separated by ultracentrifugation (Fig. 1B), a large portion of relatively lipid-soluble drugs (bepridil, verapamil and bupivacaine) is sequestered into the upper lipid layer, leading to reduced concentrations of relatively lipid-soluble drugs in the lower CAE layer and supporting the lipid sink theory $[1,10,12,13,16]$. Similar to a previous report involving verapamil (log [octanol/water partition coefficient]: $3.79)$, lipid emulsion $(0.25,0.3$ and $1 \%)$ attenuated the vasodilation caused by a toxic dose of amlodipine (Fig. 2A and B), and lipid emulsion (0.25 and 1\%) decreased amlodipine $\left(3 \times 10^{-7} \mathrm{M}\right)$ concentrations to 83 \pm 6 and $82 \pm 5 \%$ of their original concentrations, respectively (Fig. 6) [16,31]. These findings suggest that this lipid emulsion-mediated attenuation of the vasodilation induced by a toxic dose of amlodipine is associated with the reduction in amlodipine concentration caused by lipid emulsion. The attenuation of amlodipine-induced vasodilation was greater at a high concentration (1\%) of lipid emulsion than at a low concentration $(0.25 \%)$ of lipid emulsion (Fig. 2A; $P<0.05$ at 30 to $70 \mathrm{~min}$ ). However, there was no significant difference in the reduction in amlodipine concentration by low $(0.25 \%)$ and high (1\%) concentrations of lipid emulsion (Fig. 6). Thus, there may be unidentified mechanisms other than a lipid sink associated with the lipid solubility of amlodipine. On the other hand, CAE and 1\% lipid emulsion containing amlodipine did not significantly alter the reduction in amlodipine-induced vasodilation, suggesting that the lipid emulsion-mediated reduction in amlodipine-induced vasodilation may be mainly dependent on the lipid sink effect. This discrepancy (Fig. 2A, 3A and 6) may be due to physiochemical alterations in lipid emulsion containing amlodipine by the strong ultracentrifugation used to produce the lower CAE layer. As cardiovascular collapse induced by a toxic dose of amlodipine produces acidosis in vivo, further research on the effect of acidification on the lipid emulsion-mediated inhibition of vasodilation evoked by amlodipine is needed [32].

Amlodipine produces vasodilation in isolated rat aortae or pulmonary arteries precontracted with $\mathrm{KCl}$ and norepinephrine [33,34]. Amlodipine-induced vasodilation is more enhanced in $\mathrm{KCl}$-induced contraction than in norepinephrine-induced contraction [33]. Consistent with previous reports, amlodipine induced vasodilation in rat aorta precontracted with norepinephrine or $\mathrm{KCl}$ (Fig. 2C), and the magnitude of vasodilation induced by amlodipine was higher in $\mathrm{KCl}$-induced contraction than in norepinephrine-induced contraction (Fig. 2C) $[33,34]$. The contraction induced by contractile agonists such as phenylephrine involves calcium influx via the L-type calcium channels [35]. The vasodilation induced by verapamil alone was similar 
to that induced by combined treatment with verapamil and amlodipine (Fig. 3B), suggesting that the blockade of L-type calcium channels with verapamil abolishes amlodipine-induced vasodilation $[33,35]$. In contrast to $\mathrm{KCl}$-induced contraction, the contraction evoked by norepinephrine, which is released from sympathetic nerve endings, involves calcium sensitization-mediated contraction through the inhibition of myosin light chain phosphatase via Rho-kinase or PKC [36]. Norepinephrine-induced contraction was inhibited by Rho-kinase inhibitor Y-27632 or PKC inhibitor GF109203X (Fig. 4A and B), showing that norepinephrine-induced contraction is mediated by a pathway involving PKC and Rho-kinase. As amlodipine-induced vasodilation was enhanced in contraction induced by the Rho-kinase stimulant $\mathrm{NaF}$ compared with the PKC stimulant PDBu (Fig. 5A), our findings as a whole indicate that the inhibition of amlodipine-induced vasodilation by lipid emulsion (1\%) in isolated rat aortae precontracted with norepinephrine seems to rely partially on the maintenance of contraction mediated by Rho-kinase (Fig. 4A and 5A).

The limitations of this study are as follows: first, endothelial nitric oxide is involved in the regulation of vascular tone, whereas endothelium-denuded aorta pretreated with L-NAME was used in the current study to avoid compounding factors such as nitric oxide-dependent relaxation due to remaining endothelium after endothelial denudation [20,21]. Second, small resistance arterioles are the main vessels involved in peripheral vascular resistance associated with blood pressure, whereas this experiment used aortae, which are considered conduit vessels [37]. Third, a toxic dose of amlodipine causes myocardial depression, whereas this experiment did not consider the heart, which is important in controlling hemodynamics [33,38]. Fourth, the triglyceride half-life of chylomicron, which transports lipids absorbed from the intestine, was reported to be approximately 5 to $6 \mathrm{~min}$, whereas Intralipid, containing $100 \%$ long-chain triglycerides in aqueous Krebs solution, was used for approximately $70 \mathrm{~min}$ in the current experiment [39].

In conclusion, these results suggest that lipid emulsion inhibits the vasodilation induced by a toxic dose of amlodipine in isolated endothelium-denuded rat aorta by reducing the concentration of amlodipine. The vasodilation induced by a toxic dose of amlodipine in rat aortae precontracted with norepinephrine seems to be mediated mainly by blockade of the L-type calcium channel and partially by inhibition of the Rho-kinase-mediated pathway.

\section{Abbreviations}

CAE: centrifuged aqueous extract; PKC: protein kinase $\mathrm{C}$.

\section{Acknowledgments}

This research was supported by Basic Science Research Program through the National Research Foundation of Korea (NRF) funded by the Ministry of Education (NRF-2018R1D1A1B07043914). This research was supported by Basic Science Research Program through the National Research Foundation of Korea (NRF) funded by the Ministry of Education (NRF-2016R1D1A1B03930451).

\section{Competing Interests}

The authors have declared that no competing interest exists.

\section{References}

1. Weinberg GL. Lipid emulsion infusion: resuscitation for local anesthetic and other drug overdose. Anesthesiology 2012; 117: 180-7.

2. Heldens M, van der Nat GAM, Melman PG. Renal failure, shock, and loss of pacemaker capture: a case of flecainide intoxication. Neth J Med. 2019; 77: 189-92.

3. Pişkin Ö, Ayoğlu H. Effects of remifentanil pretreatment on bupivacaine cardiotoxicity in rats. Cardiovasc Toxicol. 2018; 18: 56-62.

4. French D, Armenian P, Ruan W, Wong A, Drasner K, Olson KR, Wu AH. Serum verapamil concentrations before and after Intralipid ${ }^{\circledR}$ therapy during treatment of an overdose. Clin Toxicol (Phila). 2011; 49: 340-4.

5. Liang CW, Diamond SJ, Hagg DS. Lipid rescue of massive verapamil overdose: a case report. J Med Case Rep. 2011;5:399. doi: 10.1186/1752-1947-5-399.

6. Presley JD, Chyka PA. Intravenous lipid emulsion to reverse acute drug toxicity in pediatric patients. Ann Pharmacother. 2013; 47: 735-43.

7. Sampson CS, Bedy SM. Lipid emulsion therapy given intraosseously in massive verapamil overdose. Am J Emerg Med. 2015; 33: 1844.e1. doi:10.1016/j.ajem.2015.04.061.

8. Meaney CJ, Sareh H, Hayes BD, Gonzales JP. Intravenous lipid emulsion in the management of amlodipine overdose. Hosp Pharm. 2013; 48: 848-54.

9. Patel T, Tietze D, Mehta AN. Amlodipine overdose. Proc (Bayl Univ Med Cent.) 2013; 26: 410-1.

10. Ok SH, Hong JM, Lee SH, Sohn JT. Lipid emulsion for treating local anesthetic systemic toxicity. Int J Med Sci. 2018; 15: 713-22.

11. Fettiplace MR, Weinberg G. The mechanisms underlying lipid resuscitation therapy. Reg Anesth Pain Med. 2018; 43: 138-49.

12. Hori $K$, Matsuura $T$, Tsujikawa $S$, Mori $T$, Kuno $M$, Nishikawa $K$. The significant contribution of the partitioning effect in lipid resuscitation for bupivacaine-induced cardiotoxicity: evaluation using centrifuged solution in vivo and in isolated hearts. Br J Anaesth. 2015; 115: 935-7.

13. Wagner M, Zausig YA, Ruf S, Rudakova E, Gruber M, Graf BM, Volk T. Lipid rescue reverses the bupivacaine-induced block of the fast $\mathrm{Na}+$ current (INa) in cardiomyocytes of the rat left ventricle. Anesthesiology 2014; 120: 724-36.

14. Ok SH, Shin IW, Lee SH, Park J, Woo MS, Hong JM, Kim J, Sohn JT. Lipid emulsion alleviates the vasodilation and mean blood pressure decrease induced by a toxic dose of verapamil in isolated rat aortae and an in vivo rat model. Hum Exp Toxicol. 2018; 37: 636-46.

15. Pang DC, Sperelakis N. Uptake of calcium antagonistic drugs into muscles as related to their lipid solubilities. Biochem Pharmacol. 1984; 33: 821-6.

16. Ok SH, Lee SH, Kim HJ, Hong JM, Kim JY, Bae SI, Shin Y, Sohn JT. Lipid emulsion attenuates the vasodilation induced by a toxic dose of a calcium channel blocker through its partitioning into the lipid phase. Gen Physiol Biophys. 2019; 38: 227-35.

17. NIH U.S. National Library of Medicine. Pubchem. Amlodipine. https://pubchem.ncbi.nlm.nih.gov/compound/2162\#section=Solubility (Accessed 14th June 2019)

18. Lee SH, Park CS, Ok SH, Kim D, Kim KN, Hong JM, Kim JY, Bae SI, An S, Sohn JT. Bupivacaine-induced contraction is attenuated by endothelial nitric oxide release modulated by activation of both stimulatory and inhibitory phosphorylation (Ser1177 and Thr495) of endothelial nitric oxide synthase. Eur J Pharmacol. 2019; 853: 121-8.

19. Kim SO, Toda H, Nakamura K, Miyawaki I, Hirakata H, Hirata S, Mori K. Thiopental attenuates relaxation and cyclic GMP production in vascular smooth muscle of endotoxin-treated rat aorta, independent of nitric oxide production. Br J Anaesth. 1998; 81: 601-2. 
20. Lenasi H, Kohlstedt K, Fichtlscherer B, Mülsch A, Busse R, Fleming I. Amlodipine activates the endothelial nitric oxide synthase by altering phosphorylation on Ser1177 and Thr495. Cardiovasc Res. 2003; 59: 844-53.

21. Ok SH, Lee SH, Yu J, Park J, Shin IW, Lee Y, Cho H, Choi MJ, Baik J, Hong JM, Han JY, Lee HK, Chung YK, Sohn JT. Lipid emulsion attenuates acetylcholine-induced relaxation in isolated rat aorta. Biomed Res Int. 2015;2015:871545. doi:10.1155/2015/871545.

22. Regenthal R, Krueger M, Koeppel C, Preiss R. Drug levels: therapeutic and toxic serum/plasma concentrations of common drugs. J Clin Monit Comput. 1999; 15: 529-44.

23. Karaki H, Ozaki H, Hori M, Mitsui-Saito M, Amano K, Harada K, Miyamoto S, Nakazawa H, Won KJ, Sato K. Calcium movements, distribution, and functions in smooth muscle. Pharmacol Rev. 1997; 49: 157-230.

24. Lee SH, Kang D, Ok SH, Kwon SC, Kim HJ, Kim EJ, Hong JM, Kim JY, Bae SI, An S, Sohn JT. Linoleic acid attenuates the toxic dose of bupivacaine-mediated reduction of vasodilation evoked by the activation of adenosine triphosphate-sensitive potassium channels. Int J Mol Sci. 2018;19. pii:E1876. doi: $10.3390 /$ ijms19071876.

25. Lavergne C, Martinez M, Trottier C. Empirical model selection in generalized linear mixed effects models. Computational Statistics 2008; 23: 99-109.

26. Rietjens SJ, de Lange DW, Donker DW, Meulenbelt J. Practical recommendations for calcium channel antagonist poisoning. Neth J Med. 2016; 74: 60-7.

27. Maskell KF, Ferguson NM, Bain J, Wills BK. Survival after cardiac arrest: ECMO rescue therapy after amlodipine and metoprolol overdose. Cardiovasc Toxicol. 2017; 17: 223-5

28. Weinberg GL, VadeBoncouer T, Ramaraju GA, Garcia-Amaro MF, Cwik MJ. Pretreatment or resuscitation with a lipid infusion shifts the dose-response to bupivacaine-induced asystole in rats. Anesthesiology 1998; 88: 1071-5.

29. Weinberg GL, Ripper R, Murphy P, Edelman LB, Hoffman W, Strichartz G, Feinstein DL. Lipid infusion accelerates removal of bupivacaine and recovery from bupivacaine toxicity in the isolated rat heart. Reg Anesth Pain Med. 2006; 31: 296-303

30. Ok SH, Sohn JT, Baik JS, Kim JG, Park SS, Sung HJ, Shin MK, Kwon YH, Park CS, Shin IW, Lee HK, Chung YK. Lipid emulsion reverses Levobupivacaine-induced responses in isolated rat aortic vessels. Anesthesiology 2011; 114: 293-301.

31. NIH U.S. National Library of Medicine. Pubchem. Verapamil https://pubchem.ncbi.nlm.nih.gov/compound/verapamil\#section=OctanolWater-Partition-Coefficient (Accessed 14th June 2019)

32. Ok SH, Sohn JT. Amlodipine toxicity and lipid emulsion. Korean J Anesthesiol. 2018; 71: 491-492.

33. Matlib MA, French JF, Grupp IL, Van Gorp C, Grupp G, Schwartz A. Vasodilatory action of amlodipine on rat aorta, pig coronary artery, human coronary artery, and on isolated Langendorff rat heart preparations. J Cardiovasc Pharmacol. 1988;12 Suppl 7: S50-4.

34. Woodmansey PA, Zhang F, Channer KS, Morice AH. Vasodilatory action of the calcium antagonist amlodipine on large and resistance pulmonary arteries from normoxic and chronically hypoxic rats. Clin Sci (Lond.). 1993; 85: 361-6.

35. Shin, I.W., Sohn, J.T., Kim, H.J., Kim, C., Lee, H.K., Chang, K.C., Chung, Y.K. Etomidate attenuates phenylephrine-induced contraction in isolated rat aorta. Can J Anaesth. 2005; 52: 927-34

36. Akata T. General anesthetics and vascular smooth muscle: direct actions of general anesthetics on cellular mechanisms regulating vascular tone. Anesthesiology 2007; 106: 365-91.

37. Mayet J, Hughes A. Cardiac and vascular pathophysiology in hypertension. Heart 2003; 89: 1104-9.

38. Jeppesen P, Bruun J, Nielsen-Kudsk F. Amlodipine dynamic effects and myocardial pharmacokinetics in the isolated and perfused guinea-pig heart. Pharmacol Toxicol. 1998; 82: 250-6.

39. Park Y, Damron BD, Miles JM, Harris WS. Measurement of human chylomicron triglyceride clearance with a labeled commercial lipid emulsion. Lipids 2001; 36: 115-20. 